\title{
Resistance to Soybean Cyst Nematode: Rhgl
}

\author{
Ahmed J. Afzal ${ }^{1,2,5}$, Ali Srour', Aparna Natarajan', Navinder Saini' ${ }^{2}$, M. Javed lqbal ${ }^{3}$, Matt $^{1}$ \\ Geisler ${ }^{4}$, Hany A. El Shemy ${ }^{1,2,6}$, and David A. Lightfoot ${ }^{1,2,4}$
}

'Department of Molecular Biology, Microbiology and Biochemistry, Southern Illinois University at Carbondale, IL, 62901, USA; ${ }^{2}$ Genomics Core Facility; Department of Plant Soil and Agricultural Systems, and Center for Excellence in Soybean Research, Teaching and Outreach, Southern Illinois University at Carbondale, Carbondale, IL 62901-4415, USA; ${ }^{3}$ Institute for Advanced Learning and Research (ISRR), Institute for Sustainable and Renewable Resources (IALR), 150 Slayton Ave, Danville, VA 24540, USA; ${ }^{4}$ Department of Plant Biology, Southern Illinois University at Carbondale, IL, 62901, USA; ${ }^{5}$ Present address: Department of Horticulture and Crop Science, 2021 Coffey Rd, Ohio State University, Columbus, OH, 43210, USA; ${ }^{6}$ Present address: Faculty of Agriculture Research Park (FARP) and Department of Biochemistry, Faculty of Agriculture, Cairo University, 12613 Giza, Egypt.

Received: October 10, 2011 / Accepted: February 12, 2012

\section{Abstract}

The genes underlying rhg 1 lie at a sometimes dominant sometimes co-dominant locus, necessary for resistance to all Hg types of the soybean (Glycine max (L.) Merr.) cyst nematode (Heterodera glycines). Genomic research identified; nucleotide changes within a candidate gene encoding a receptor like kinase (RLK) that were capable of altering root development and thereby part of the resistance to $\mathrm{Hg}$ types $\mathrm{O}$ (race 3); changes in a laccase that are capable of altering cyst development; and genes underlying changes in membrane biology. This set of three genes are subject to co-selection with a modifier locus on another linkage block. Root development is slowed in the resistant seedling and results in end of season yield loss when SCN is not present. However, in the presence of $\mathrm{SCN}$ resistant seedling roots grow just as vigorously as the now slower growing parasitized susceptible roots and therefore show little loss to SCN parasitism. In some genotypes but not others the RLK can act alone to confer resistance. Functional paralogs of the three gene cluster have been found on other linkage groups including A1, B1, G, and 0 and these can be functional in different sources of resistance like G. soja, PI 437654 and PI438489B. At rhgl the allele differences change the structure, interacting partners and activity of the LRR protein and the laccase. The changes between the alleles result in about 30 other proteins (judged by 2 D gels), 112 metabolites (by FTICRMS) and 8 metabolites (by
GCMS) to increase in abundance in roots during $S C N$ infection in the resistant NILs. Understanding the basis of root stunting by resistance alleles will be used to improve methods for developing new nematode resistant soybean cultivars that do not suffer from the yield suppression and low seed germination rates of existing cultivars.

Keywords: Soybean cyst nematode (SCN), nematode resistance, proteomics, soybean roots.

\section{Introduction}

Soybean [Glycine max (L.) Merr.] is the world's most important legume crop, it is grown for both oil and protein co-products. Many natural by-products are also derived from soybean and several possess significant antimicrobial and pharmacological properties (Dixon and Sumner, 2003). The abundance and variety of soybean's bioactive factors suggest the species has evolved and been domesticated in the presence of many persistent pathogen populations.

After water deficit, the leading causes of seed yield losses in soybean are biotic stresses from pathogen infections (Wrather et al., 2001). Amongst soybean pathogens, the soybean cyst nematode ( $\mathrm{SCN}$ ) causes the most damage. Yield losses in the 
world due to SCN infection have approached $\$ 2$ billion a year (Niblack et al., 2004) and in the US alone amounted to 1.5 billion for the 1996-1997 period (Chen et al., 2001). SCN preventative measures include crop rotation, the use of resistant cultivars and nematicides. The use of resistant cultivars provides the most efficient and practical control of SCN.

Soybean breeding has used heavily three plant introductions (PIs) resistant to the cyst nematode (Brucker et al., 2005b). The $\mathrm{Pls}$ 'PI88788' 'Peking' and 'PI437654' have each been shown to carry resistance loci effective against multiple nematode races. However, the resistance to $\mathrm{SCN}$ is quantitative and controlled by multiple loci. Previously identified were two quantitative trait loci that provided substantial resistance against the soybean cyst nematode $\mathrm{Hg}$ Type $\mathrm{O}$ (a biotype of race 3 ) in NILs derived from Peking via 'Forrest' (Meksem et al., 2001; Ruben et al., 2006). The resistance locus Rhg 4 on linkage group A2 provided resistance against $\mathrm{Hg}$ Type 0 whereas rhg 1 , the resistance locus on linkage group $G$ provided some resistance against nematodes of all known races. The rhg 1 locus allele from Peking causes a rapid necrosis of the giant cells and the formation of thickened cell walls around the defunct feeding site (syncitium) by $24-48 \mathrm{~h}$ after the SCN female starts to feed, some 4-5 days after infestation of the roots (Alkharouf et al., 2004, 2006; Ithal et al., 2007a,b). The activity of the resistance allele is associated with global changes in transcript abundance (TA) within the syncitium and systemic changes in the root system with hundreds of transcripts altered. However, in plants the correlations between transcript, protein and metabolite abundances are low. Therefore, key proteins may have been overlooked by transcript analysis. Further, some proteins can alter the metabolome without alterations in their abundances (Edwards, 1996; Ito et al., 2003; Schad et al., 2005; Mungur, 2008). Therefore, key metabolites may remain undetected. Finally, protein-protein and protein-metabolite interactions are important in many plant responses to the environment (Geisler-Lee et al., 2008). Therefore, key interactions may remain undetected by the transcript analyses reported to date.

The rhg 1 locus appears to be a multifunctional and multigeneic locus (Ruben et al., 2006; lqbal et al., 2009; Afzal et al., 2009a). The locus contained several genes capable of altering the transcriptome, proteome, metabolome and interactome including; a receptor like kinase inferred to alter root development (RLK; Afzal and Lightfoot, 2007; Afzal et al., 2008a,b; 2009a); an unusual laccase involved in phenolic metabolism (lqbal et al., 2009); and a predicted $\mathrm{Na} / \mathrm{H}$ antiporter. Flanking the locus were two proteins of unknown function, one of which is transcribed in roots (Triwitayakorn et al., 2005; Ruben et al., 2006). Each of the genes was encoded different alleles with significant amino acid and nucleotide changes in resistant soybean compared to susceptible soybean (Ruben et al., 2006; Afzal et al., 2008b; 2009a). Analysis in near isogeneic lines suggests the resistance alleles of the core set of three linked genes cannot be separated by recombination without the loss of resistance (Afzal, 2007; Afzal et al., 2008b; 2009a). Key elements of that hypothesis are reviewed in this paper.

\section{Materials and Methods}

\section{Plant Materials}

The genetic materials and methods used were described in Triwitayakorn et al. (2005), Ruben et al. (2006), Afzal et al. (2008; 2009a,b, 2012). Briefly, the seeds of RILs and the NIL populations derived from the cross of Essex by Forrest were obtained from Dr. Paul Gibson at Southern Illinois University at Carbondale in 1995 and were increased from 1995 to present at the Agronomy Research Center (Lightfoot et al., 2005). NIL populations were developed and maintained as described in Triwitayakorn et al. (2005). Genetic identity and purity were checked after increase and before each experiment with 5-10 SSR markers/ line and DNA from 5-10 seeds/line. Seeds of NIL 34-23 (resistant haplotype between markers Satt 214 to Satt 570) NIL34-33 (heterogeneous and heterozygous across the interval) and NIL 34-3 (susceptible haplotype across the interval) were used at the F5:13 generation.

Seedlings were grown in a growth chamber and growth was analyzed as described by Afzal et al. (2012). DNA extractions and marker analysis also followed the methods of Afzal et al. (2012).

\section{Results}

\section{Dominant, Recessive or Co-dominant Nature of rhgl}

Both recessive and co dominant roles have been assigned for the rhg 1 locus. In both past and recent studies with PIs, resistance encoded by rhg 1 was reported as recessive (Arelli et al., 1990; Brucker et al., 2005b) whereas previously in NILs, the rhg 1 locus was reported to be co-dominant (Meksem et al., 2001a) but without single plant marker allele associations. Clearly, co-dominant and recessive roles of plant disease resistance loci are rare and unusual (Li et al., 2006; Nieto et al., 2007). On the basis of the segregation pattern at the intrageneic TMD1 (intron) and Sca5 (promoter) markers, in NIL 34-33 background, the rhg 1 locus was shown to be dominant (Figure 1; Table 1).

In NIL 34-33 segregating at the rhg 1 locus, 4 plants were heterozygous at the TMD1 and Sac5 markers. The cyst scores, for all plants in the NIL population corresponded with the respective alleles at the rhg 1 locus. For plants polymorphic at TMD1, the cyst score correspond to those for resistant plants. Therefore, the rhg 1 locus was dominant in this set of NILs. Genotypes were rhg 1 -e/rhg 1 -e Rhg 4-p/Rhg 4-p for NIL 34-3 and Rhg $1-p /$ Rhg 1 -p Rhg 4-p/Rhg4-p for NIL 34-23 whereas NIL34-33 contained both those and Rhg $1-p / r h g l-e ~ R h g 4-p / R h g 4-p$ in different plants. The discrepancies in dominance among different populations may be associated with the genetic background the gene resides in and may result from interactions among genes at the rhg 1 locus and/or modifier genes at other loci (Webb et al., 1995). 
Table 1. Cosegregation of alleles of the marker TMDI in the RLK at rhg 1 and co-dominant resistance to $\mathrm{SCN}$.

\begin{tabular}{|l|l|l|l|}
\hline Plant/line & Score & Cyst No. & Phenotype \\
\hline 12 & A/B & 012 & Resistant/Heterozygous \\
\hline 13 & A & 224 & Susceptible \\
\hline 14 & A & 177 & Susceptible \\
\hline 15 & A/B & 026 & Resistant/Heterozygous \\
\hline 16 & B & 000 & Resistant \\
\hline 17 & A & 209 & Susceptible \\
\hline 18 & B & 014 & Resistant* \\
\hline 19 & A & 187 & Susceptible* \\
\hline 20 & A/B & 020 & Resistant/Heterozygous \\
\hline 21 & B & 021 & Resistant \\
\hline 22 & A & 176 & Susceptible \\
\hline
\end{tabular}

(A)

\section{$\begin{array}{llllllllllll}12 & 13 & 14 & 15 & 16 & 17 & 19 & 18 & 20 & 21 & 22\end{array}$}

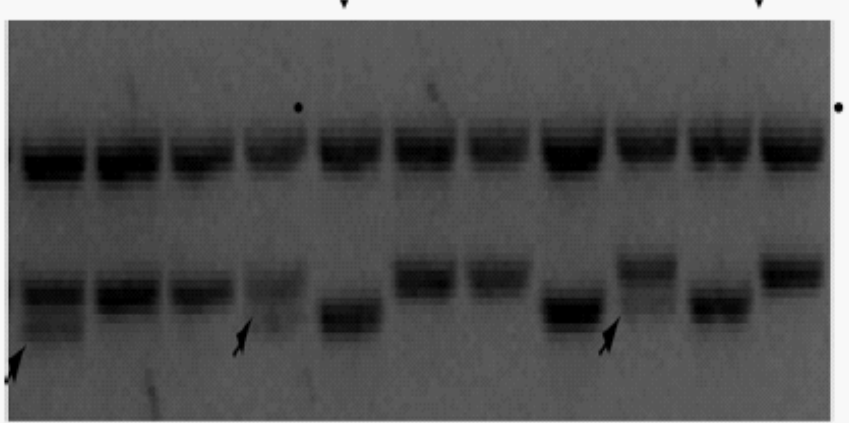

The recombination events found among the six $\mathrm{Hg}$ Type 0 susceptble Pls (Ruben et al., 2006) suggests that the action of rhg 1 requires elements to the distal side of the RLK intron, possibly one or all of the 3 polymorphisms found in the intracellular kinase of the complete RLK. Some mutations in the kinases of other plant RLKs are known to be lethal (Lease et al., 2001; Wang et al., 2006). Kinase mutants can be lethal in many cases (Sessa et al., 2000; Kollewe et al. 2004). Therefore, it may be the kinase at the rhg 1 locus that underlies restricted root growth in resistant genotypes directly or after some sort of interaction.

\section{Syntenic Paralogs of the rhgl Locus}

To identify rhg 1 paralogs, primers specific to the conserved regions of rhgl leucine-rich repeat and rhg 1 - kinase domain were designed and the genome sequence examined. $\mathrm{H} 38 \mathrm{f} 23$ was on Lg B 1 and scaffold 139 (69100-144000). Significantly, this BAC and scaffold 139 contained a complete set of syntenic genes for a second rhg 1 locus (the RLK, laccase both antiport-

(B)

Figure 1. Analysis of NIL 34-33 with TMD1 and other markers. Panel A; Out of the 34 plants analyzed in this run (10 shown), 4 were heterozygous (Lanes 12, 14, 20 and 29). The four heterozygous plants had a resistant phenotype (arrowed). The upper invariant band is from the RLK paralog on LGO, the lower band is from the intron of the RLK on LG G. SCN counts and gel scores for the plants are given in Table 1. Plants 18 and 19 were mislabeled. Panel B Marker map of the genomic region around rhgl showing TMD1 position and locus ideogramshowing the locations of genes.

\section{Inhibition of Root Growth by Alleles of rhgl in the NILs}

When counting the cysts with prior knowledge of the allele it was noted that root mass and vigor appeared to differ among genotypes. Measurements of root mass showed a significant difference among NILs that was associated with the allele at rhg 1 or linked loci (Figure 2; Table 3). Across several experiments, both NILs that were pure breeding susceptible and NILs that segregated some susceptible lines had higher root masses than their SCN resistant counterparts. This phenomenon might underlie the global association of resistance to SCN with low seed germination, seedling vigor, stand formation and ultimately seed yield. ers, the kinase and the helicase; Figure 3). The DNA markers sequence paralogs were present but more diverged. Sequence analysis of the alleles in Williams 82 and Forrest RLK at rhg 1 showed $99 \%$ amino sequence identity. Among the paralogs DNA sequence identity was high ( $\sim 92 \%$ in geneic region; Supplemental Figure 1). Amino acid identity was $84 \%$ in the LRR, $86 \%$ in the transmembrane domain and $94 \%$ in the kinase domain. The laccase and the anitported also shoed $85-96 \%$ amino acid identity.

Both of the RLK paralogs were located by BLAT of BES to sequence scaffolds and were in regions where loci with functions similar to rhgl were located Lg Al (the RLK; Vierling et al., 1997) and LG B1 (the syntenic cluster; Wu et al., 2009). 
A.
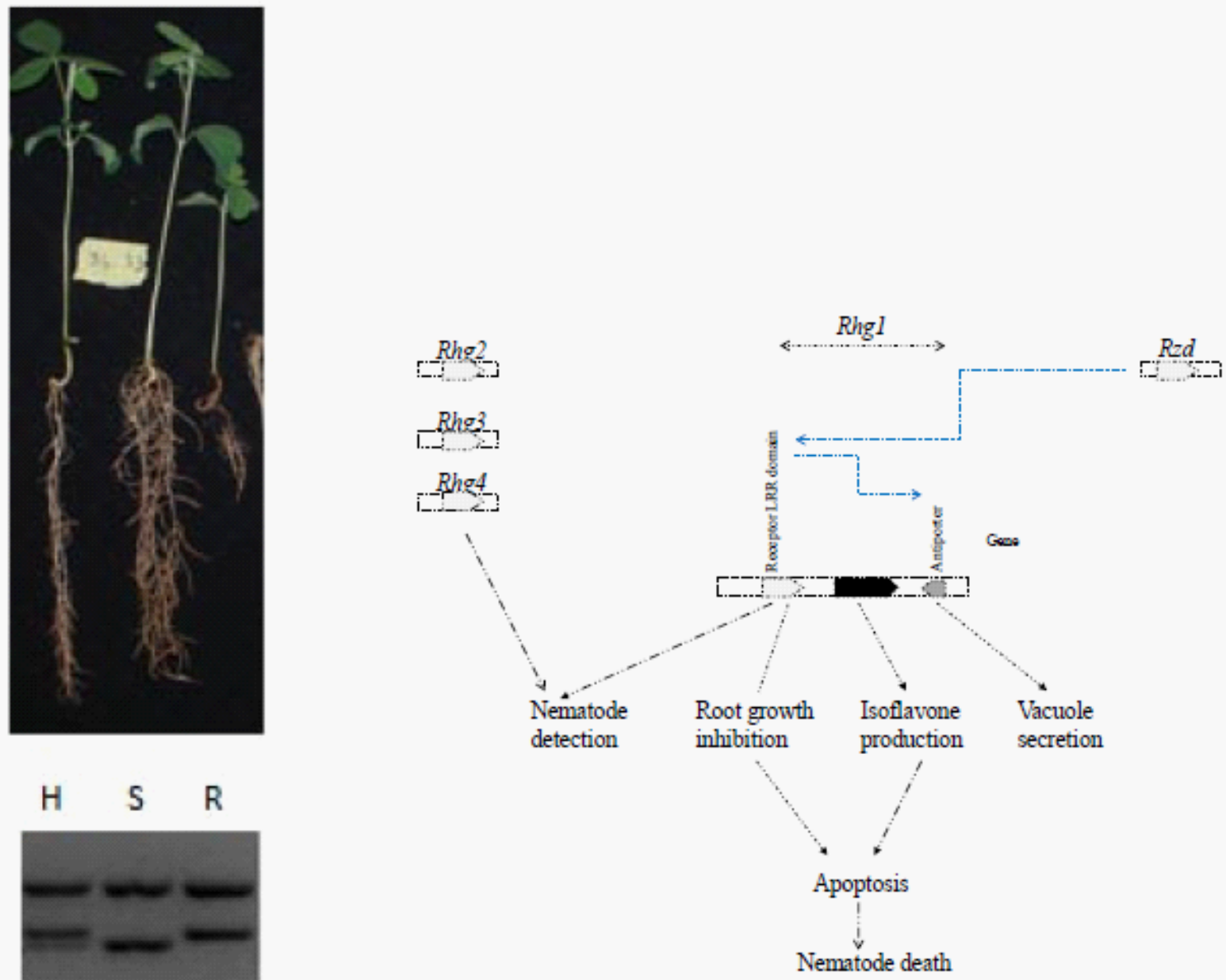

C.

Figure 2. The RHG 1 protein alters root development. Panel $A_{\text {; }}$ Soybean NILS at 2 weeks after planting but pre SCN inoculation show different root morphologies, post inoculation root masses are not different (by 6 weeks). Therefore. rhg 1 inhibits germination and early root growth. Panel $B$; the root morphologies cosegregated with the allele in the RLK at the rhg 1 locus as shown by the intragenic marker TMD 1 (satellite in the intron). Panel $C_{;}$Model for the function of the rhg 1 locus in resistance to $\mathrm{SCN}$. Black arrows show positive interactions, blue arrows show inhibitions. In this model four primary phenotypic events are controlled by the 3 genes at rhg 1 and four unlinked genes Rhg 2-4 and Rzd 1 (sup-Rhg 1 ). The apoptosis cause in the giant cell of the nematodes feeding sites will occur in plant root cells in the absence of Rzdl allele in the coupled phase with the RLK. Root growth inhibition occurs despite suppression of the RLK.

The paralogs may encode proteins that recognize novel race biotypes or substitute for rhgl following activation in certain Pls or crosses.

\section{Examining the rhgl Interactome}

Two dimensional gel electrophoresis that resolved more than 1,000 protein spots on each gel showed rhg 1 controlled the abundance of only thirty (Afzal et al., 2009b). The proteins were grouped into six functional categories. Metabolite analysis by GC-MS identified 131 metabolites among which 58 were altered by one or more treatment, 28 were involved in primary metabolisms. Taken together the data showed seventeen pathways that were altered by rhg 1 controlled metabolisms associated with SAR-like responses including xenobiotic, phytoalexin, ascorbate and inositol metabolism as well as primary metabo- lisms like amino acid metabolism and glycolysis. The pathways impacted by the rhg 1 allelic state and SCN infestation agreed with transcript abundance analyses but identified a smaller set of key proteins.

Six of the proteins lay within the same region of the interactome identifying a key set of 159 proteins inferred to be involved in nuclear protein transport and degradation. The predicted interactome showed the chaperonin, Fl-ATPase, multi catalytic endopeptidase, thaumatin-like protein, cytosolic heat shock protein and triose phosphate isomerase were interacting indirectly through 2 to 7 intermediates (Figure 4). The cluster of all the potential interacting neighbors showed 159 proteins in total, with 194 interactions. The protein functions included transcription factors, chaperonins, signal transduction factors and metabolisms involved in energy generation (Table 1). The four proteins in the cluster with most interacting partners were a GTP 


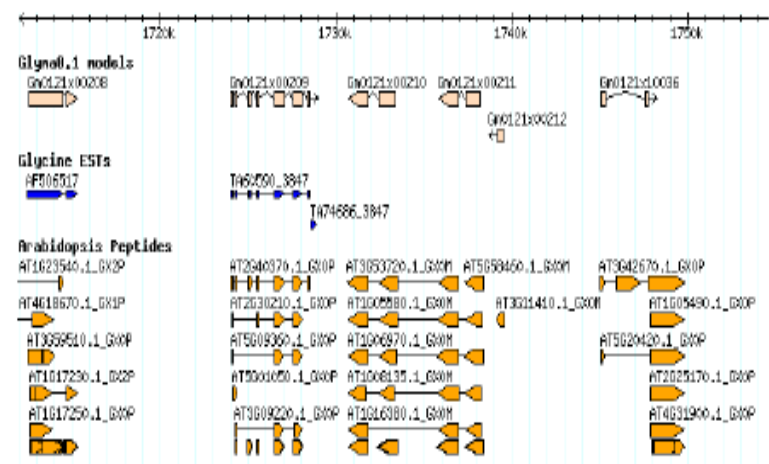

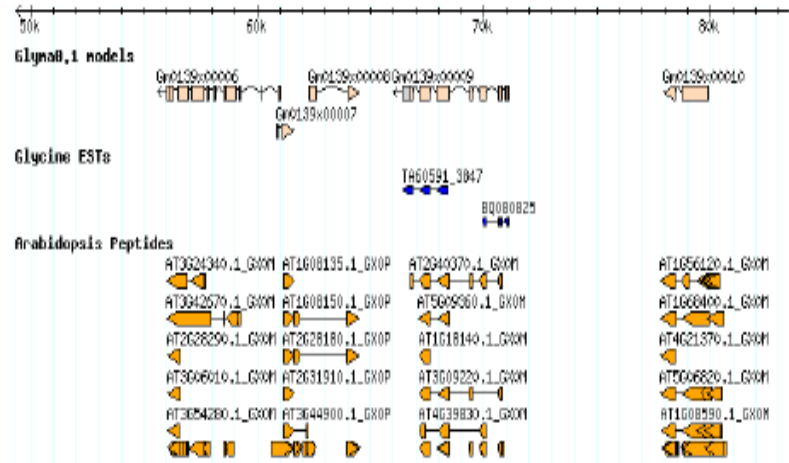

Figure 3. GBrowse view of $40 \mathrm{Mbp}$ encompassing the rhg 1 locus from $\mathrm{Lg} G$ and the syntenic gene cluster from $\operatorname{LgB} 1$ in Williams 82. The image was viewed at Phytosome.net in 2008. Panel A shows the region around rhg 1 on scaffold 121 (LgG). Panel B shows the region around the rhg 1 paralog on scaffold 139 ( $L g$ B 1 ) Note gene orders are the same but the scaffold orientation is inverted. Panel $C$ shows the alignment of the two DNA sequences and Panel D the amino acid aligments centered on the RLK at rhg 1 .

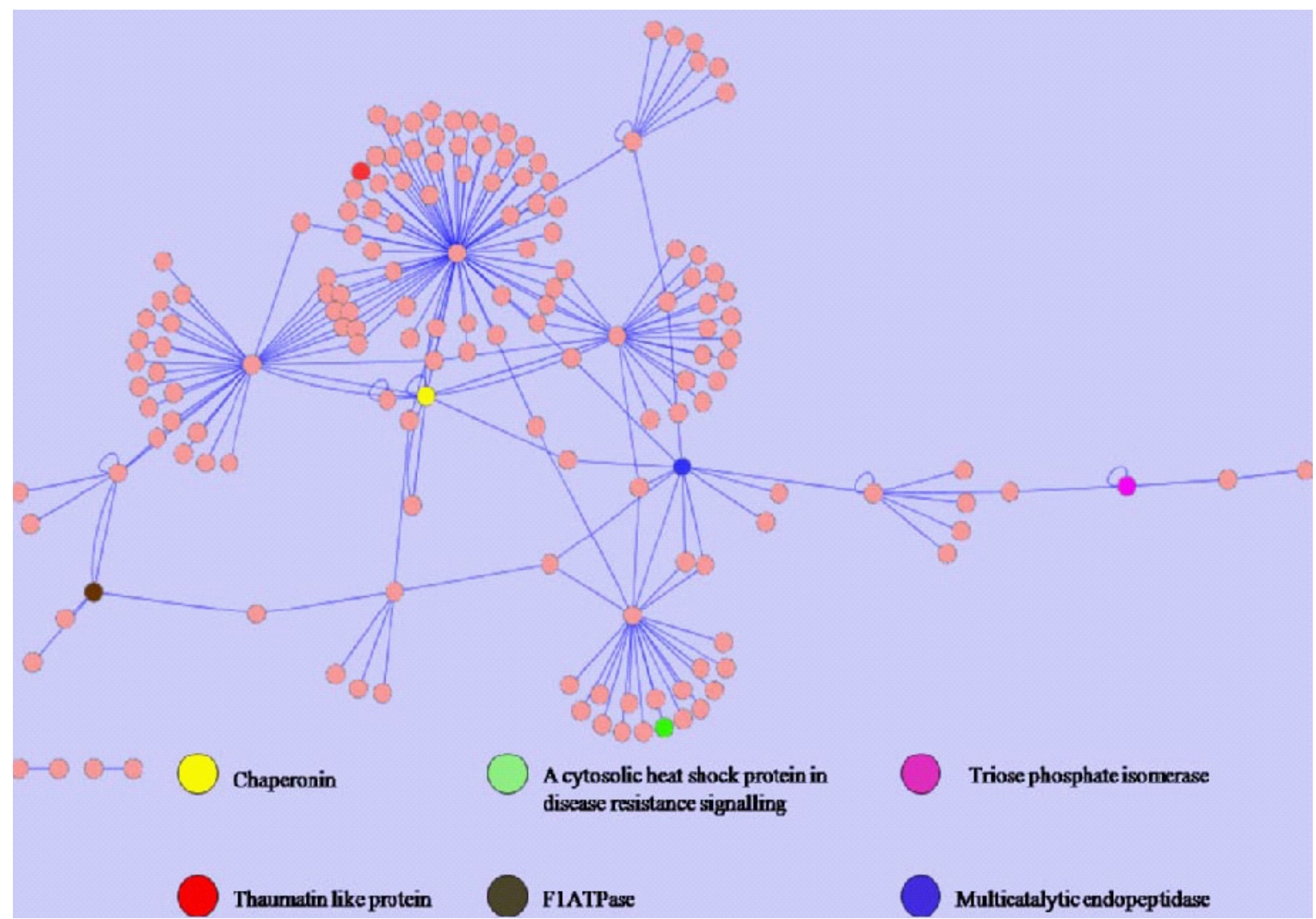

Figure 4. A part of the predicted interactome for soybean showing six proteins from the 2D gel analysis (chaperonin, F1ATPase, multi catalytic endopeptidase, thaumatin like protein, cytosolic heat shock protein and triose phosphate isomerase). The interactome was extended by one node to include 159 nodes (proteins) and 194 edges (interactions). Two proteins with a previously characterized role in disease resistance were identified within this part of the interactome; the thaumatin like protein induced in response to fungal pathogens (blue) and a cytosolic heat shock protein in disease resistance signaling (green). All six proteins interacted through 2-7 intermediate partners (see Supplemental Table 1 Afzal et al. (2009b) for pink spot identification numbers). Auto-regulation was predicted for the triose phosphate isomearse and the chaperonin. 
binding-family protein (28 partners), importin alpha- 2 subunit (64 partners), importin beta-2 subunit (22 partners) and a $26 \mathrm{~S}$ proteasome non-ATPase regulatory subunit $3 a$ (21 partners). Therefore, the regulation of protein degradation and transport of proteins across the nuclear membranes may be important aspects of resistance to $\mathrm{SCN}$.

Finally, two proteins (glucose 6 phosphate isomerase, EC 5.3.1.9; and isoflavone reductase, EC 1.3.1.45) and two metabolites (maltose and unidentified) differed in resistant and susceptible NILs without SCN infestation and may formthe basis of a new assay for the selection of resistance to $\mathrm{SCN}$ in soybean.

\section{Conclusions}

The data presented here suggests the genes linked to the primary candidate RLK may encode factors involved in the modulation of rhg 1 activity. Possible roles include contributions to additive resistance; contributions to resistance in other resistance types (eg PI88788 and Toyohazu; R types 2 and 3) or contributions to the resistance to other $\mathrm{Hg}$ types (Nelsen et al., 2003). Unlikely, in view of the susceptibility of segregation events within the interval from the RLK to Satt309 in both PI evolution and NIL segregation, is the hypothesis that the linked genes are factors necessary in susceptible genotypes for SCN parasitism. The dominance of rhgl in NIL segregation also suggests the genes are active in resistant types and inactive in susceptible genotypes. In conclusion, the rhg 1 locus was inferred to be a complex of from three to several genes assembled and coinherited over long periods of selection for resistance to an pandemic pest, root parasitic nematodes. The genes in the loci were shown to regulate pathways involved in protein trafficking, protein degradation and primary metabolisms. Transgenic plants will be used to tease apart the layered functions in the next phase of this research.

\section{References}

Afzal AJ and DA Lightfoot (2007) Soybean disease resistance protein RHG 1-LRR domain expressed, purified and refolded from Escherichia coli inclusion bodies: preparation for a functional analysis. Protein Expr Purif 53: 346-355.

Afzal AJ (2007) Structure-function analysis of a candidate receptor like kinase protein in soybean cyst nematode resistance and identification of accessory proteins involved in plant defense. PhD Dissertation. Southern Illinois University, Carbondale.

Afzal AJ, AJ Wood, and DA Lightfoot (2008a) Plant Receptor-Like Serine Threonine Kinases: Roles in Signaling and Plant Defense. Molec Plant Microb Interact 21:507-517.

Afzal AJ, N Saini, A Srour, and DA Lightfoot (2008b) The multigeneic rhg 1 locus: a model for the effects on root development, nematode resistance and recombination suppression. Nature Preceed hdl: 10101 /npre.2008.2726.1. online).

Afzal AJ, N Saini, A Srour, and DA Lightfoot (2009a) The multigeneic rhg 1 locus complete sequence identifies alleles of three genes underlying root development, nematode resistance and recombination suppression. Nature Genet. in review).

Afzal AJ, A Natarajan, N Saini, MJ Iqbal, MA Geisler, H El Shemy, R Mungur, L Willmitzer, and DA Lightfoot (2009) The nematode resistance allele at the rhg 1 locus alters the proteome and metabolome of soybean roots. Plant Physiology. in press).

Afzal AJ, R Mungur, L Wilmitzer, and DA Lightfoot (2009c) The metabolite fingerprint of the rhg 1 Locus in Soybean Roots. J Biomed Biotech 2009: in review).

Afzal AJ, A Srour, N Hemmati, N Saini, H El Shemy, and DA Lightfoot (2012) Recombination suppression at the dominant Rhg $1 / R f s 2$ locus underlying soybean resistance to the cyst nematode. Theor Appl Genet 124: 1027-1039.

Alkharouf N, R Khan, and B Matthews (2004) Analysis of expressed sequence tags from roots of resistant soybean infected by the soybean cyst nematode. Genome 47: 380-388.

Alkharouf NW, VP Klink, IB Chouikha, HS Beard, MH MacDonald, S Meyer, HT Knap, R Khan, and BF Matthews (2006) Time-course microarray analyses reveal global changes in gene expression of susceptible Glycine max. soybean roots during infection by Heterodera glycines (soybean cyst nematode). Planta 224: 838-852.

Arelli PR (1994) Inheritance of resistance to Heterodera glycines race 3 in soybean accessions. Plant Dis 78: 898-900.

Brucker E, S Carlson, E Wright, T Niblack, and B Diers (2005) Rhgl alleles from soybean PI 437654 and PI 88788 respond differentially to isolates of Heterodera glycines in the greenhouse. Theor Appl Genet 111 : 44-49.

Dixon RA and LW Sumner (2003) Legume natural products: Understanding and manipulating complex pathways for human and animal health. Plant Physiol 131: 878-885.

Geisler-Lee J, N O'Toole, R Ammar, NJ Provart, AH Millar, and MA Geisler (2008) Predicted Interactome for Arabidopsis thaliana. Plant Physiol 145: 317-329.

lqbal MJ, R Ahsan, AJ Afzal, A Jamai, K Meksem, H El Shemy, and DA Lightfoot (2009) Analysis of the activity of the soybean laccase encoded within the Rfs $2 /$ rhg 1 locus. Current Issues in Molecular Biology i1 1: 11-19.

Ithal N, J Recknor, D Nettleton, L Hearne, T Maier, TJ Baum, and MG Mitchum (2007a) Parallel genome-wide expression profiling of host and pathogen during soybean cyst nematode infection of soybean. Mol Plant Microbe Interact 20:293-305.

Ithal N, J Recknor, D Nettleton, T Maier, TJ Baum, and MG Mitchum (2007b) Developmental transcript profiling of cyst nematode feeding cells in soybean roots. Mol Plant Microbe Interact 20: 510-525.

Klink VP, N Alkharouf, M MacDonald, and B Matthews (2005) Laser capture microdissection. LCM. and expression analyses of Glycine max. soybean. syncytium containing root regions formed by the plant pathogen Heterodera glycines. soybean cyst nematode). Plant Mol Biol 59: 965-979.

Klink VP, CC Overall, NW Alkharouf, MH Macdonald, and BF Matthews (2007a) Laser capture microdissection. LCM. and comparative microarray expression analysis of syncytial cells isolated from incompatible and compatible soybean. Glycine max roots infected by the soybean cyst nematode (Heterodera glycines). Planta 226: 1389-1409.

Klink VP, CC Overall, NW Alkharouf, MH Macdonald, and BF Matthews (2007b) A time-course comparative microarray analysis of an incompatible and compatible response by Glycine max. soybean to Heterodera glycines (soybean cyst nematode). infection. Planta 226: 1423-1447.

Kollewe C, AC Mackensen, D Neumann, J Knop, P Cao, S Li, H Wesche, MU Martin (2004) Sequential auto-phosphorylation steps in the interleukin- 1 receptor-associated kinase- 1 regulate its availability as an adapter in interleukin-1 signaling. J Biol Chem 279: 5227-5236.

Lease KA, NY Lau, RA Schuster, KU Torii, and JC Walker (2001) Receptor serine/threonine protein kinases in signalling: analysis of the erecta receptor-like kinase of Arabidopsis thaliana. New Phytol 151: 133-143. 
Meksem K, P Pantazopoulos, VN Nijiti, DL Hyten, PR Arelli, and DA Lightfoot (2001) 'Forrest' resistance to the soybean cyst nematode is bigenic: saturation mapping of the Rhg 1 and Rhg 4 loci. Theor Appl Genet 103: 710-717.

Mungur R (2008) Characterisation of florigenic signals between plant systems: a tissue-specific and spatio-temporal analysis. Verlag $\mathrm{Dr}$ Mueller. VDM. Press, Saarbrucken, Germany.

Mysore KS, MD D'Ascenzo, X He, and GB Martin (2003) Over-expression of the disease resistance gene Pto in tomato induces gene expression changes similar to immune responses in human and fruitfly. Plant Physiol 132: 1901-1912.

Puthoff DP, D Nettleton, SR Rodermel, and TJ Baum (2003) Arabidopsis gene expression changes during cyst nematode parasitism showed by statistical analyses of microarray expression profiles. Plant J 33: $911-921$.

Ruben E, J Aziz, AJ Afzal, VN Niiti, K Triwitayakorn, MJ lqbal, S Yaegashi, P Arelli, C Town, K Meksem, and DA Lightfoot (2006) Genomic analysis of the 'Peking' rhg 1 locus: candidate genes that underlie soybean resistance to the cyst nematode. Molec Genet Genom 276: 320-330.

Sessa G, M D'Ascenzo, and GB Martin (2000) Thr38 and Ser198 are Pto auto-phosphorylation sites required for the AvrPto-Pto-mediated hypersensitive response. EMBO J 19: 2257-2269.

Srour A, AJ Afzal, N Saini, L Blahut-Beatty, N Hemmati, DH Simmonds,
H El Shemy, CD Town, H Sharma, X Liu, W Li, and DA Lightfoot (2012) The receptor like kinase transgene from the Rhg1/Rfs2 locus caused pleiotropic resistances to soybean cyst nematode and sudden death syndrome. BMC Genomics (In Review).

Triwitayakorn K, VN Niiti, MJ Iqbal, S Yaegashi, CD Town, and DA Lightfoot (2005) Genomic analysis of a region encompassing QRfs 1 and QRfs2: Genes that underlie soybean resistance to sudden death syndrome. Genome 48: 125-138.

Vierling RA, Faghihi J, Ferris VR, Ferris JM. 1996. Association of RFLP markers conferring broad-based resistance to the soybean cyst nematode. Heterodera glycines). Theor Appl Genet 92: 83-86.

Wang X, MB Goshe, EJ Soderblom, BS Phinney, JA Kuchar, J Li, T Asami, $S$ Yoshida, SC Huber, and SD Clouse (2005) Identification and functional analysis of in vivo phosphorylation sites of the Arabidopsis BRASSINOSTEROID-INSENSITIVE 1 receptor kinase. Plant Cell 17 1685-1703.

Webb DM, BM Baltazar, PR Arelli, J Schupp, K Clayton, P Keim, and WD Beavis (1995) Genetic mapping of soybean cyst nematode race-3 resistance loci in the soybean PI 437.654. Theor Appl Genet 91: 574-581.

Wu X, S Blake, D Sleper, G Shannon, P Cregan, and H Nguyen (2009) QTL, additive and epistatic effects for SCN resistance in PI 437654. Theor Appl Genet 118 (6): 1093-1105. 